\title{
Connection Failure Detection Mechanism of UMTS Charging Protocol
}

\author{
Hui-Nien Hung, Yi-Bing Lin, Fellow, IEEE, Nan-Fu Peng, and Sok-Ian Sou
}

\begin{abstract}
In Universal Mobile Telecommunications System (UMTS), the extension of GPRS tunneling protocol called GTP' is utilized to transfer the Charging Data Records (CDRs) from GPRS Support Nodes (GSNs) to Charging Gateways (CGs). To ensure that the mobile operator receives the charging information, availability for the GTP' transmission is essential. One important issue on GTP' availability is connection failure detection. It is desirable to select appropriate parameter values to avoid false failure detections (e.g., temporary network congestions) while to detect the true failures quickly. We propose an analytic model to compute the false failure detection probability and the expected true failure detection time. Based on our study, the network operator can select the appropriate parameter values for various traffic conditions to reduce the probability of false failure detection and/or true failure detection time.
\end{abstract}

Index Terms-GPRS Tunneling Protocol extension (GTP'), charging protocol, connection failure detection, Charging Data Record (CDR).

\section{INTRODUCTION}

$\mathbf{U}$ NIVERSAL Mobile Telecommunications System (UMTS) [1], [7] supports high-speed Packet Switched (PS) data for accessing versatile multimedia services. The PS Core Network is an IP-based backbone network [8]. This core network consists of GPRS Support Nodes (GSNs) such as Serving GSNs (SGSNs) and Gateway GSNs (GGSNs). The Charging Gateway (CG) collects the billing and charging information from the GSNs. The GTP' protocol [3] is utilized to transfer the Charging Data Records (CDRs) from GSNs to CGs. When a Mobile Station is receiving a UMTS PS service, the CDRs are generated based on the charging characteristics (data volume limit, duration limit and so on) of the subscription information for that service. A CG analyzes and possibly consolidates the CDRs from various GSNs, and passes the consolidated data to a billing system.

A CG maintains a GSN list. An entry in the list represents a GTP' connection to a GSN. This entry consists of pointers to a $C D R$ database and the sequence numbers of possibly duplicated packets. A GSN maintains a list of CGs in the priority

Manuscript received May 13, 2004; revised December 22, 2004; accepted March 27, 2005. The associate editor coordinating the review of this letter and approving it for publication was Z. Zhang. This work was sponsored in part by NSC Excellence project NSC93-2752-E-0090005-PAE, ITRI/NCTU Joint Research Center, and IIS/Academia Sinica. The work of H.-N. Hung was supported in part by the National Science Council of Taiwan under Grant NSC-92-2118-M-009-013.

H.-N. Hung and N.-F. Peng are with the Institute of Statistics, National Chiao Tung University, Hsinchu 30010, Taiwan, R.O.C. (e-mail: hhung@stat.nctu.edu.tw; nanfu@stat.nctu.edu.tw).

Y.-B. Lin and S.-I. Sou are with the Department of Computer Science and Information Engineering, National Chiao Tung University, Hsinchu 30010, Taiwan, R.O.C. (e-mail: liny@csie.nctu.edu.tw; sisou@csie.nctu.edu.tw).

Digital Object Identifier 10.1109/TWC.2006.05026. order (typically ranges from 1 to 100). If a GSN unexpectedly loses its connection to the current CG, it may send the CDRs to the next CG in the priority list. An entry in the CG list describes parameters for GTP' transmission. After sending a GTP' request, a GSN may not receive a response from the CG due to network failure, network congestion or temporary node unavailability. In this case, 3GPP TS 29.060 [2] defines a mechanism for request retry, where the GSN will retransmit the message until either a response is received within a timeout period or the number of a retry threshold is reached. In the latter case, the GSN-CG communication link is considered disconnected. This paper studies the availability issues for GTP'. Specifically we propose an analytic model to investigate the GTP' connection failure detection mechanism. Our study will provide guidelines for the mobile operators to select the parameters for GTP' connection manipulation.

\section{GTP' Failure Detection Mechanism}

This section describes the Path Failure Detection Algorithm (PFDA) that detects path failure between the GSN and the CG. In a GSN, an entry in the CG list represents a GTP' connection to a CG. We describe the entry attributes related to PFDA as follow:

- The $C G$ address attribute identifies the CG connected to the GSN.

- The Status attribute indicates if the connection is "active" or "inactive".

- The Charging Packet Ack Wait Time $\left(T_{r}\right)$ is the maximum elapsed time the GSN is allowed to wait for the acknowledgement of a charging packet; typical allowed values range from 1 millisecond to 65 seconds.

- The Maximum Number of Charging Packet Tries $(L)$ is the number of attempts (including the first attempt and the retries) the GSN is allowed to send a charging packet; typical $L$ range is $1-16$. When $L=1$, it means that there is no retry.

- The Maximum Number of Unsuccessful Deliveries $(K)$ is the maximum number of consecutive failed deliveries that are attempted before the GSN considers a connection failure occurs. Note that a delivery is considered failed (or timed out if it has been attempted for $L$ times without receiving any acknowledgement from the CG).

- The Unsuccessful Delivery Counter $\left(N_{K}\right)$ attribute records the number of the consecutive failed delivery attempts.

- The Unacknowledged Buffer stores a copy of each GTP' message that has been sent to the CG but has not been acknowledged. A record in the unacknowledged 
buffer consists of an Expiry Timestamp $t_{e}$, the Charging Packet Try Counter $\left(N_{L}\right)$ and an unacknowledged GTP' message. The expiry timestamp $t_{e}$ is equal to $T_{r}$ plus the time when the GTP' message was sent, which represents the expiry of the message. The counter $N_{L}$ counts the number of the first attempt and retries that have been performed for this charging packet transmission.

PFDA works as follows:

Step 1. After the connection setup procedure is complete, both $N_{L}$ and $N_{K}$ are set to 0 , and the Status is set to "active". At this point, the GSN can send GTP' messages to the $\mathrm{CG}$.

Step 2. When a GTP' message is sent from the GSN to the CG at time $t$, a copy of the message is stored in the unacknowledged buffer, where the expiry timestamp is set to $t_{e}=t+T_{r}$.

Step 3. If the GSN has received the acknowledgement from the CG before $t_{e}$, both $N_{L}$ and $N_{K}$ are set to 0 .

Step 4. If the GSN has not received the acknowledgement from the CG before $t_{e}, N_{L}$ is incremented by 1 . If $N_{L}=$ $L$, then the charging packet delivery is considered failed. $N_{K}$ is incremented by 1 .

Step 5. If $N_{K}=K$, then the GTP' connection is considered failed. The Status is set to "inactive".

When Step 5 of PFDA is encountered, it is assumed that the path between the GSN and the $\mathrm{CG}$ is no longer available, and the GSN is switched to another CG. However, besides link failure, unacknowledged packet transfers may also be caused by temporary network congestion. In this case, it is not desirable to perform CG switching (which is a very expensive operation). A simple way to avoid this kind of "false" failure detection is to set large values for parameters $T_{r}, L$ and $K$. On the other hand, large parameter values may result in delayed detection of "true" failures. Therefore, it is important to select appropriate parameter values so that true failures can be quickly detected while false failures can be avoided. Based on the GTP' mechanism described in this section, we derive the probability of false failure detection in Section III, and compute the expected detection time of true failure in Section IV.

\section{Probability of False Failure Detection}

Let random variable $t_{f}$ be the lifetime between when the GTP' connection is established and when a true failure occurs. During this period, undesirable false failures (temporary network congestions) may be detected, and the GSN is unnecessarily switched to another CG. Let $\alpha$ be the probability that the PFDA detects a false failure (and therefore the GSN is switched to another CG before a true failure occurs). Suppose that $t_{f}$ has the density function $f_{f}\left(t_{f}\right)$. Let the arrivals of charging packets be a Poisson stream with rate $\lambda_{c}$. Note that the charging packets delivered between a GSN and the CG are generated by all users in this GSN. Each CDR stream of an individual user may have an arbitrary distribution, but the net traffic of all users becomes a Poisson stream [11]. We observe that the charging packets forms a Poisson stream when there are more than 20 users. Let the Echo message arrivals be a deterministic stream with the fixed interval $T_{e}$.
For any reasonable setting, an Echo message should not be issued before the previous one is acknowledged or timed out. Thus, in CG configuration, we set

$$
T_{e} \geq L T_{r}
$$

Let random variable $N_{c}\left(t_{f}\right)$ be the number of charging packet arrivals (excluding retries) during the lifetime $t_{f}$ of the GTP' connection. Then

$$
\operatorname{Pr}\left[N_{c}\left(t_{f}\right)=n\right]=\left[\frac{\left(\lambda_{c} t_{f}\right)^{n}}{n !}\right] e^{-\lambda_{c} t_{f}}
$$

Let random variable $N_{e}\left(t_{f}\right)$ denote the number of Echo message arrivals (excluding retries) during $t_{f}$. That is

$$
N_{e}\left(t_{f}\right)=\left\lfloor t_{f} / T_{e}\right\rfloor
$$

Let $N\left(t_{f}\right)$ be the number of GTP' messages (excluding retries) that the GSN attempts to deliver to the CG during $t_{f}$. That is, $N\left(t_{f}\right)=N_{e}\left(t_{f}\right)+N_{c}\left(t_{f}\right)$. From (3), $N\left(t_{f}\right)=\left\lfloor t_{f} / T_{e}\right\rfloor+$ $N_{c}\left(t_{f}\right)$. Therefore, for a given $t_{f}$, (2) can be re-written as

$$
\operatorname{Pr}\left[N\left(t_{f}\right)=\left\lfloor t_{f} / T_{e}\right\rfloor+n\right]=\left[\frac{\left(\lambda_{c} t_{f}\right)^{n}}{n !}\right] e^{-\lambda_{c} t_{f}}
$$

Let random variable $t_{r}$ be the round-trip transmission delay (between the GSN and the CG) for a GTP' message attempt. We assume that $t_{r}$ has a distribution $F_{r}\left(t_{r}\right)$ and the density function $f_{r}\left(t_{r}\right)$. From Step 4 of PFDA, a transmission is timed out with probability $\operatorname{Pr}\left[t_{r} \geq T_{r}\right]$. From Step 5 of PFDA, a delivery is timed out (after it has been tried for $L$ times) with probability $p$, where

$$
p=\left(\operatorname{Pr}\left[t_{r} \geq T_{r}\right]\right)^{L}=\left[1-F_{r}\left(T_{r}\right)\right]^{L}
$$

The GTP' connection is considered disconnected after $K$ consecutive delivery timeouts where each of the deliveries fails for $L$ attempts (see Step 5 of PFDA). Since the GTP' path is connected during $t_{f}$, a false failure is detected if Step 5 of PFDA is executed when the $j$-th GTP' message delivery is timed out, where $j \leq N\left(t_{f}\right)$. Let $\theta(j)$ denote the probability that such false failure is detected at the $j$-th delivery. Assume that the delivery results (i.e., a success or a failure) are independent. Based on the relationship between $j$ and $K, \theta(j)$ is derived in three cases:

Case I. $0 \leq j<K$. It is clear that $\theta(j)=0$.

Case II. $j=K$. It is clear that $\theta(j)=p^{K}$.

Case III. $j>K$. In this case, no false failure is detected before the $(j-K-1)$-th delivery, the $(j-K)$-th delivery is a success, and the last $K$ deliveries are timed out. Therefore, $\theta(j)=\left[1-\sum_{i=0}^{j-K-1} \theta(i)\right](1-p) p^{K}$.

From (5) and the three cases described above, we have

$\theta(j)= \begin{cases}0 & , 0 \leq j<K \\ p^{K} & , j=K \\ {\left[1-\sum_{i=0}^{j-K-1} \theta(i)\right](1-p) p^{K}} & , j>K\end{cases}$

For $K=1$ and $j \geq 1$, (6) is simplified as $\theta(j)=(1-p)^{j-1} p$. In this case, $\theta(j)$ becomes a geometric distribution. Let $\bar{\theta}(j)$ be the probability that no false failure is detected before (and including) the $j$-th GTP' message delivery. Then

$$
\bar{\theta}(j)=1-\sum_{i=0}^{j} \theta(i)
$$


From (4) and (7), the probability $\alpha$ of false failure detection is

$$
\begin{aligned}
\alpha= & 1-\int_{t_{f}=0}^{\infty} \sum_{n=0}^{\infty} \bar{\theta}\left(\left\lfloor t_{f} / T_{e}\right\rfloor+n\right) \\
& \times \operatorname{Pr}\left[N\left(t_{f}\right)=\left\lfloor t_{f} / T_{e}\right\rfloor+n\right] f_{f}\left(t_{f}\right) d t_{f}
\end{aligned}
$$

The derivation for (8) can be extended by assuming that the lifetime $t_{f}$ has an exponential distribution with rate $\lambda_{f}$. The exponential distribution is chosen because it has often been used in reliability and lifetime modeling [10]. We note that our result can be easily generalized for $t_{f}$ with mixed-Erlang distribution with a tedious routine. Eq. (8) is re-written as

$$
\begin{aligned}
\alpha= & 1-\lambda_{f} \sum_{k=0}^{\infty} \sum_{n=0}^{\infty} \bar{\theta}(k+n)\left[\frac{\lambda_{c}^{n}}{\left(\lambda_{c}+\lambda_{f}\right)^{n+1}}\right] \\
& \times \sum_{j=0}^{n}\left\{\frac{e^{-\left(\lambda_{c}+\lambda_{f}\right) k T_{e}}\left[\left(\lambda_{c}+\lambda_{f}\right) T_{e}\right]^{j}}{j !}\right\} \\
& \times\left[k^{j}-e^{-\left(\lambda_{c}+\lambda_{f}\right) T_{e}}(k+1)^{j}\right]
\end{aligned}
$$

\section{Expected True Failure Detection Time}

This section derives the expected detection time of "true" failure. Consider the timing diagram in Fig. 1(a), where a failure occurs at time $t_{f}$ and is detected at time $t_{d}$. The detection time for the failure is $\tau_{d}=t_{d}-t_{f}$. Let random variable $N_{K}(t)$ represent the $N_{K}$ value at time $t$. If $N_{K}\left(t_{f}\right)=$ $K-n$ (for $0<n \leq K$ ), then the GTP' connection failure is detected when $n$ more GTP' message deliveries are timed out. Consider a GTP' message sent from the GSN to the CG. The GSN either receives an acknowledgement from the CG or the delivery (i.e., the $L$-th transmission for this message) is timed out at time $t^{*}$. This time $t^{*}$ is denoted as the departure time of the GTP' message delivery. For $1 \leq i \leq n$, let $t_{d, i}$ be the departure time of the $i$-th failed GTP' message delivery after $t_{f}$. Note that $t_{d}=t_{d, n}$. In Fig. 1(b), the arrival times $t_{a, i}$ (for $1 \leq i \leq n$ ) correspond to the GTP' message deliveries with the departure times $t_{d, i}$ in Fig. 1(a). It is apparent that $t_{a, i}=t_{d, i}-L T_{r}$. Note that these arrivals may occur before or after $t_{f}$. In Fig. 1(b), the first $j$, deliveries arrive before $t_{f}$. If

$$
t_{a, n}>t_{f}
$$

then the true failure detection time $\tau_{d}$ is

$$
\tau_{d}=t_{d, n}-t_{f}=t_{a, n}+L T_{r}-t_{f}
$$

In this section, we compute the probability that $N_{K}\left(t_{f}\right)=$ $K-n$ (for $0<n \leq K$ ). This probability is used to derive $E\left[\tau_{d} \mid t_{a, n}>t_{f}\right]$. Then $E\left[\tau_{d}\right]$ is computed from $E\left[\tau_{d} \mid t_{a, n}>\right.$ $\left.t_{f}\right]$ derived in the following subsections and $E\left[\tau_{d} \mid t_{a, n} \leq t_{f}\right]$ derived in [12].

\section{A. Derivation for the $N_{K}\left(t_{f}\right)$ distribution}

We first compute $\operatorname{Pr}\left[N_{K}\left(t_{f}\right)=0\right]$. Then we use this result to derive $\operatorname{Pr}\left[N_{K}\left(t_{f}\right)=j\right]$ (for $1 \leq j \leq K-1$ ). It is clear that $t_{f}$ lies in two consecutive Echo message arrivals. Suppose that these two Echo messages arrive at times $t_{0}$ and $t_{0}+T_{e}$, respectively (see Fig. 2). Since $t_{f}$ is a random observer, it is uniformly distributed over $\left[t_{0}, t_{0}+T_{e}\right)$. Let random variable $N_{K \rightarrow \infty}(t)$ be the $N_{K}$ value at time $t$ when $K \rightarrow \infty$. In interval $\left[t_{0}, t_{0}+T_{e}\right),\left\{N_{K \rightarrow \infty}(t) ; t \in\left[t_{0}, t_{0}+T_{e}\right)\right\}$ is a continuous time, discrete state stochastic process (the state space is $0,1,2, \ldots)$. There exists $j$ such that for $1 \leq i \leq j$ the interval $\left[t_{0}, t_{0}+T_{e}\right)$ consists of $j$ alternative periods $\left(x_{i}, y_{i}\right)$, where

$$
N_{K \rightarrow \infty}(t) \begin{cases}=0 & , \text { for } t \text { in one of the } x_{i} \text { periods } \\ >0 & , \text { for } t \text { in one of the } y_{i} \text { periods }\end{cases}
$$

If $N_{K \rightarrow \infty}\left(t_{0}\right) \neq 0$, then $x_{1}=0$. Similarly, if $N_{K \rightarrow \infty}\left(t_{0}+T_{e}\right)=0$, then $y_{j}=0$. Let $X=\sum_{i=1}^{j} x_{i}$ and $Y=\sum_{i=1}^{j} y_{i}$. Then

$$
\operatorname{Pr}\left[N_{K \rightarrow \infty}(t)=0\right]=\frac{E[X]}{E[X]+E[Y]}=\frac{E[X]}{T_{e}}
$$

From (12), $\operatorname{Pr}\left[N_{K \rightarrow \infty}(t)=j\right]$ (for $j>0$ ) is expressed as

$$
\operatorname{Pr}\left[N_{K \rightarrow \infty}(t)=j\right]=(1-p) p^{j-1}\left(1-E[X] / T_{e}\right)
$$

In (13), the last GTP' message arrival before $t$ is timed out with probability $\left(1-E[X] / T_{e}\right)$, and the probability that there are exact $j-1$ delivery timeouts before this last GTP' message delivery is $(1-p) p^{j-1}$. Suppose that no false failure is detected before $t_{f}$. Under this condition, $N_{K}\left(t_{f}\right)$ ranges from 0 to $K-1$. From (12) and (13), we have

$\operatorname{Pr}\left[N_{K}\left(t_{f}\right)=j\right]= \begin{cases}\frac{E[X]}{T_{e}-p^{K-1}\left(T_{e}-E[X]\right)} & , j=0 \\ \frac{(1-p) p^{j-1}\left(T_{e}-E[X]\right)}{T_{e}-p^{K-1}\left(T_{e}-E[X]\right)} & , 0<j<K\end{cases}$

In (14), $E[X]$ is derived as follows. Let $t_{l}\left(0<t_{l} \leq L T_{r}\right)$ be the delivery delay for a GTP' message delivery (including retries). In Fig. $2, k>0$ departures occur in $\left[t_{0}, t_{0}+T_{e}\right)$, where the $i$-th departure occurs at $t_{i}$ (for $1 \leq i \leq k$ ). Let $t_{k+1}=t_{0}+T_{e}$ be the arrival time of the next Echo message. According to (1), the departure of the previous Echo message must occur in $\left(t_{0}, t_{0}+T_{e}\right)$. Suppose that this departure is the $j$ th departure where $j \leq k$. By considering whether the previous Echo message delivery fails or successes, we express $E[X]$ as

$$
\begin{aligned}
E[X]= & E\left[X \mid t_{l}=L T_{r}\right] \operatorname{Pr}\left[t_{l}=L T_{r}\right] \\
& +E\left[X \mid t_{l}<L T_{r}\right] \operatorname{Pr}\left[t_{l}<L T_{r}\right]
\end{aligned}
$$

$E\left[X \mid t_{l}=L T_{r}\right]$ is derived as follows. When $t_{l}=L T_{r}$, the previous Echo message delivery fails. That is, $t_{j}=t_{0}+L T_{r}$ and $N_{K \rightarrow \infty}\left(t_{j}\right) \neq 0$. Let $z_{i}=t_{i+1}-t_{i}$ for $0 \leq i \leq k$. Since the $N_{K}$ value is only changed at times when departures occur, $z_{i}$ contributes to $E\left[X \mid t_{l}=L T_{r}\right]$ if $N_{K \rightarrow \infty}\left(t_{i}\right)=0$. Let $C=\operatorname{Pr}\left[N_{K \rightarrow \infty}\left(t_{0}\right)=0\right]$. For $j \leq k$, we have

$$
\begin{aligned}
E\left[X \mid t_{l}=L T_{r}\right]= & (1-p)\left\{E\left[\sum_{i=0}^{j-1} z_{i}\right]+E\left[\sum_{i=j+1}^{k} z_{i}\right]\right\} \\
& +C E\left[z_{0}\right]
\end{aligned}
$$

Since $\sum_{i=1}^{j-1} z_{i}=L T_{r}-z_{0}$ and $\sum_{i=j+1}^{k} z_{i}=T_{e}-L T_{r}-z_{j}$, (16) is re-written as

$$
\begin{aligned}
& E\left[X \mid t_{l}=L T_{r}\right] \\
= & (1-p)\left(T_{e}-E\left[z_{j}\right]\right)+(C+p-1) E\left[z_{0}\right]
\end{aligned}
$$

In (17), $C=\operatorname{Pr}\left[N_{K \rightarrow \infty}\left(t_{0}\right)=0\right]$ is derived in [12]. $E\left[z_{0}\right]$ is derived as follows. If the first charging packet departure 


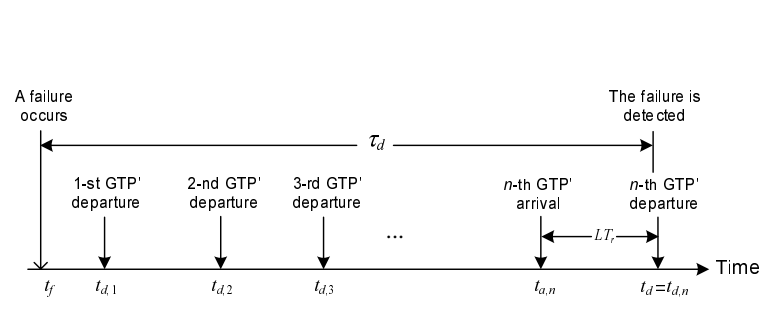

(a) Departures after a true failure

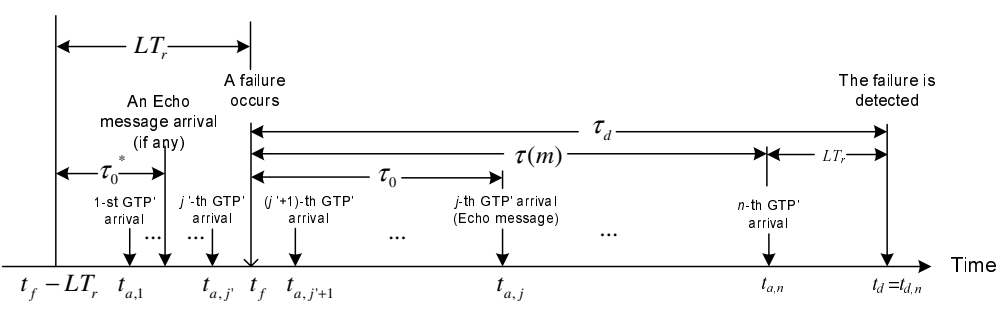

(b) Arrivals corresponding to the departures in (a) where $t_{a, n}>t_{f}$

Fig. 1. Timing Diagram for Detecting True Failure $(n \leq K)$

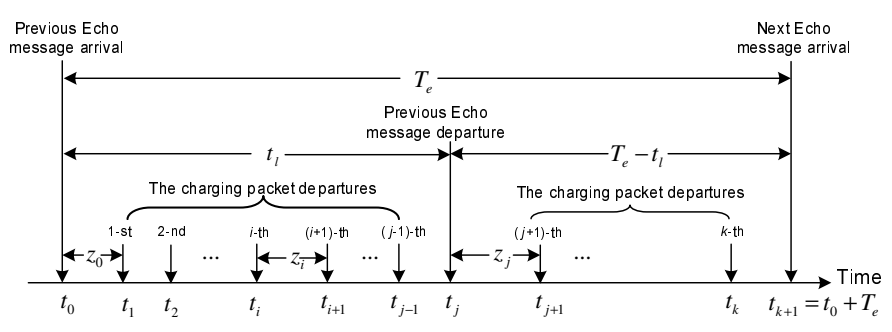

Fig. 2. Timing Diagram for Deriving $E[X]$

occurs before $t_{0}+L T_{r}$, then $z_{0}$ is exponentially distributed under the condition that $z_{0}<L T_{r}$. That is

$$
\begin{aligned}
& E\left[z_{0} \mid z_{0}<L T_{r}\right] \operatorname{Pr}\left[z_{0}<L T_{r}\right] \\
= & \frac{1}{\lambda_{c}}\left(1-e^{-\lambda_{c} L T_{r}}\right)-L T_{r} e^{-\lambda_{c} L T_{r}}
\end{aligned}
$$

If the first charging packet departure occurs after $t_{0}+L T_{r}$, then $z_{0}=L T_{r}$. In this case

$$
E\left[z_{0} \mid z_{0}=L T_{r}\right] \operatorname{Pr}\left[z_{0}=L T_{r}\right]=L T_{r} e^{-\lambda_{c} L T_{r}}
$$

Combining (18) and (19) to yield

$$
E\left[z_{0}\right]=\frac{1}{\lambda_{c}}\left(1-e^{-\lambda_{c} L T_{r}}\right)
$$

Following similar derivation, $E\left[z_{j}\right]$ can be expressed as

$$
E\left[z_{j}\right]=\frac{1}{\lambda_{c}}\left[1-e^{-\lambda_{c}\left(T_{e}-L T_{r}\right)}\right]
$$

From (17), (20) and (21), we have

$$
\begin{aligned}
& E\left[X \mid t_{l}=L T_{r}\right] \operatorname{Pr}\left[t_{l}=L T_{r}\right] \\
= & p\left\{(1-p) T_{e}+\frac{1}{\lambda_{c}}\left\{(C+p-1)\left(1-e^{-\lambda_{c} L T_{r}}\right)\right.\right. \\
& \left.\left.-(1-p)\left[1-e^{-\lambda_{c}\left(T_{e}-L T_{r}\right)}\right]\right\}\right\}
\end{aligned}
$$

$E\left[X \mid t_{l}<L T_{r}\right]$ is derived as follows. When $0<t_{l}<L T_{r}$, the previous Echo message delivery successes. That is, $t_{j}=$ $t_{0}+t_{l}<t_{0}+L T_{r}$ and $N_{K \rightarrow \infty}\left(t_{j}\right)=0$. Let $z_{i}\left(t_{l}\right)$ be the $z_{i}$ value for a specific $t_{l}<L T_{r}$. Then for $t_{l}<L T_{r}$,

$$
\begin{aligned}
E\left[X \mid t_{l}\right]= & (1-p)\left\{E\left[\sum_{i=1}^{j-1} z_{i}\left(t_{l}\right)\right]+E\left[\sum_{i=j+1}^{k} z_{i}\left(t_{l}\right)\right]\right\} \\
& +C E\left[z_{0}\left(t_{l}\right)\right]+E\left[z_{j}\left(t_{l}\right)\right]
\end{aligned}
$$

Following similar derivation for (22), for $t_{l}<L T_{r}$,

$$
\begin{aligned}
E\left[X \mid t_{l}\right]= & \frac{1}{\lambda_{c}}\left\{(C+2 p-1)-(C+p-1) e^{-\lambda_{c} t_{l}}\right. \\
& \left.-p e^{-\lambda_{c} T_{e}} e^{\lambda_{c} t_{l}}\right\}+(1-p) T_{e}
\end{aligned}
$$

Suppose that $t_{l}$ has the density function $f_{l}\left(t_{l}\right)$ and the distribution function $F_{l}\left(t_{l}\right)$. If the previous Echo message is successfully delivered, the delivery delay is $0<t_{l}<L T_{r}$ with probability $f_{l}\left(t_{l}\right) d t_{l}$. Therefore,

$$
E\left[X \mid t_{l}<L T_{r}\right] \operatorname{Pr}\left[t_{l}<L T_{r}\right]=\int_{t_{l}=0}^{L T_{r}} E\left[X \mid t_{l}\right] f_{l}\left(t_{l}\right) d t_{l}
$$

where $E\left[X \mid t_{l}\right]$ is expressed in (24), and $f_{l}\left(t_{l}\right)$ is derived in [12]. Then $E[X]$ can be obtained from (15), (22) and (25). Finally, $\operatorname{Pr}\left[N_{K}\left(t_{f}\right)=j\right]$ can be computed by using (14) and (15).

\section{B. Derivation for $E\left[\tau_{d}\right]$}

For $t_{a, n}>t_{f}$, let $m>0$ denote the number of failed GTP' message arrivals occurring after $t_{f}$. Note that $m$ is not necessarily equal to $K-N_{K}\left(t_{f}\right)$ because some GTP' message arrivals may occur before $t_{f}$ and are timed out after $t_{f}$. Such messages are denoted as cross messages ("cross" means that the delivery delay "crosses" the time point $t_{f}$ ). Therefore, the departures of cross messages are not accurately counted in $N_{K}\left(t_{f}\right)$. Fortunately, we know that these departures must occur by $t_{f}+L T_{r}$, and therefore $m=K-N_{K}\left(t_{f}+L T_{r}\right)$. $N_{K}\left(t_{f}+L T_{r}\right)$ can be derived from $N_{K}\left(t_{f}\right)$ as follows. Let $n_{c}$ and $n_{e}$ denote the numbers of cross charging packets and cross Echo messages, respectively (in Fig. 1(b); $j^{\prime}=n_{c}+n_{e}$ ). It can be observed that

$$
N_{K}\left(t_{f}+L T_{r}\right)=\min \left\{N_{K}\left(t_{f}\right)+n_{c}+n_{e}, K\right\}
$$

Note that when $m=K-N_{K}\left(t_{f}+L T_{r}\right)=0$, we have $t_{a, n} \leq t_{f}$. In this special case, $m=0$ and $E\left[\tau_{d} \mid m=0\right]$ is derived in [12]. Now assume that $m>0$. Since the deliveries of charging packets can be modeled by the $\mathrm{M} / \mathrm{G} / \infty$ system and $t_{f}$ is a random observer of the system, $n_{c}$ can be represented by a Poisson random variable with parameter $\rho$ (see Chapter 2.4 in [9]), where

$$
\rho=\lambda_{c} \int_{t_{l}=0}^{L T_{r}}\left[1-F_{L}\left(t_{l}\right)\right] d t_{l}
$$

and the probability mass function of $n_{c}$ is given by

$$
\operatorname{Pr}\left[n_{c}=i\right]=\left(\frac{\rho^{i}}{i !}\right) e^{-\rho}
$$

In Fig. 1(b), let $t_{a, j}$ (for $n_{c}+n_{e}<j$ ) be the arrival time of the first Echo message occurring after $t_{f}$, and $\tau_{0}=t_{a, j}-t_{f}$. Since $T_{e} \geq L T_{r}$, the $n_{e}$ value is either 0 or 1 . Let $\operatorname{Pr}\left[n_{e}=\right.$ 
$\left.{ }_{1} \mid \tau_{0}\right]$ be the probability that $n_{e}=1$ for a specific $\tau_{0}$. Then $\operatorname{Pr}\left[n_{e}=1 \mid \tau_{0}\right]$ can be expressed as

$\operatorname{Pr}\left[n_{e}=1 \mid \tau_{0}\right]= \begin{cases}0 & , \tau_{0} \leq T_{e}-L T_{r} \\ 1-F_{L}\left(T_{e}-\tau_{0}\right) & , \tau_{0}>T_{e}-L T_{r}\end{cases}$

where $F_{L}(t)$ is derived in [12]. In (29), when $\tau_{0} \leq T_{e}-$ $L T_{r}$, there is no undelivered Echo message before $t_{f}$. When $\tau_{0}>T_{e}-L T_{r}$, an Echo message arrival occurs in period $\left[t_{f}-L T_{r}, t_{f}\right)$. This Echo message delivery fails before $t_{f}$ with probability $\operatorname{Pr}\left[n_{e}=1 \mid \tau_{0}\right]=1-F_{L}\left(T_{e}-\tau_{0}\right)$. From (28) and (29), $\operatorname{Pr}\left[n_{c}+n_{e}=j^{\prime} \mid \tau_{0}\right]$ can be expressed as

$$
= \begin{cases}\operatorname{Pr}\left[n_{c}+n_{e}=j^{\prime} \mid \tau_{0}\right] \\
\left\{\begin{aligned}
e^{-\rho}\left(1-\operatorname{Pr}\left[n_{e}=1 \mid \tau_{0}\right]\right) & , j^{\prime}=0 \\
e^{-\rho}\left[\frac{\rho^{j^{\prime}}-1}{\left(j^{\prime}-1\right) !}\right] \operatorname{Pr}\left[n_{e}=1 \mid \tau_{0}\right] & \\
+\frac{\rho^{j^{\prime}}}{j^{\prime} !}\left(1-\operatorname{Pr}\left[n_{e}=1 \mid \tau_{0}\right]\right) & , j^{\prime}>0
\end{aligned}\right.\end{cases}
$$

Therefore, for $i \leq j<K, \operatorname{Pr}\left[N_{K}\left(t_{f}+L T_{r}\right)=j \mid \tau_{0}\right]$ can be computed from $\operatorname{Pr}\left[N_{K}\left(t_{f}\right)=i\right]$ and (30) as

$$
\begin{aligned}
& \operatorname{Pr}\left[N_{K}\left(t_{f}+L T_{r}\right)=j \mid \tau_{0}\right] \\
= & \sum_{i=0}^{j} \operatorname{Pr}\left[N_{K}\left(t_{f}\right)=i\right] \operatorname{Pr}\left[n_{c}+n_{e}=j-i \mid \tau_{0}\right]
\end{aligned}
$$

For $m>0$, let $\tau(m)=t_{a, n}-t_{f}$ (see Fig. 1(b)). $E[\tau(m)]$ is derived as follows. Let $m_{c}$ and $m_{e}$ denote the numbers of charging packet arrivals and Echo message arrivals occurring in period $\tau(m)$. That is, $m=m_{c}+m_{e}=n-\left(n_{c}+n_{e}\right)>0$. We have

$$
m_{e}=\left\lfloor\left(\tau(m)-\tau_{0}\right) / T_{e}\right\rfloor+1
$$

If $\tau_{0}>\tau(m)$, then $m_{e}=0$. Let $\tau_{e}$ be the interval between $t_{f}$ and the arrival time of the $m_{e}$-th Echo message after $t_{f}$. By convention, $\tau_{e}=0$ for $m_{e}=0$. Let $\tau_{c}$ be the interval between $t_{f}$ and the arrival time of the $m_{c}$-th charging packet after $t_{f}$. Then $\tau(m)=\max \left\{\tau_{c}, \tau_{e}\right\}$. Note that $m_{e}$ is determined by $\tau(m)$ and $\tau_{0}$ (see (32)), and therefore $\tau_{e}$ and $\tau_{c}$ are dependent of each other. Since the arrivals of charging packets are a Poisson stream, $\tau_{c}$ has the Erlang distribution with mean $m_{c} / \lambda_{c}$ and shape parameter $m_{c}$. For $m>0$, the distribution function $F_{c}\left(\tau_{c}\right)$ of $\tau_{c}$ is

$$
F_{c}\left(\tau_{c}\right)=1-\sum_{i=0}^{m_{c}-1}\left[\frac{\left(\lambda_{c} \tau_{c}\right)^{i}}{i !}\right] e^{-\lambda_{c} \tau_{c}}
$$

For $m>0$, let $F_{m}(\tau(m))$ be the distribution function of $\tau(m)$. From (32) and (33), we have

$$
\begin{aligned}
& F_{m}\left(\tau(m) \mid \tau_{0}\right)=F_{c}\left(\tau(m) \mid \tau_{0}\right) \\
= & 1-\sum_{i=0}^{m-\left\lfloor\frac{\left(\tau(m)-\tau_{0}\right)}{T_{e}}\right\rfloor-2}\left\{\frac{\left[\lambda_{c} \tau(m)\right]^{i}}{i !}\right\} e^{-\lambda_{c} \tau(m)}(34)
\end{aligned}
$$

Note that $F_{m}\left(\tau(m) \mid \tau_{0}\right)$ is discontinuous at points $\tau(m)=$ $\tau_{0}+j T_{e}$, for $j=0,1, \ldots, m_{e}-1$. From (34) we have

$$
\begin{aligned}
& \operatorname{Pr}\left[\tau(m)=\tau_{0}+j T_{e} \mid \tau_{0}\right] \\
= & F_{m}\left(\tau_{0}+j T_{e} \mid \tau_{0}\right)-F_{m}\left(\tau_{0}+j T_{e}^{-} \mid \tau_{0}\right) \\
= & \left\{\frac{\left[\lambda_{c}\left(\tau_{0}+j T_{e}\right)\right]^{m-j-1}}{(m-j-1) !}\right\} e^{-\lambda_{c}\left(\tau_{0}+j T_{e}\right)}
\end{aligned}
$$

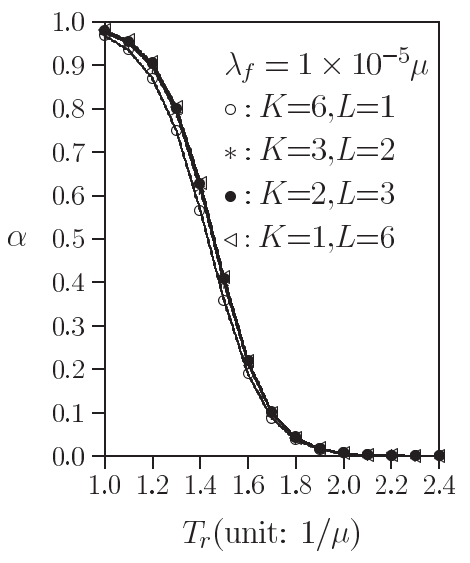

(a) Effect of $L$

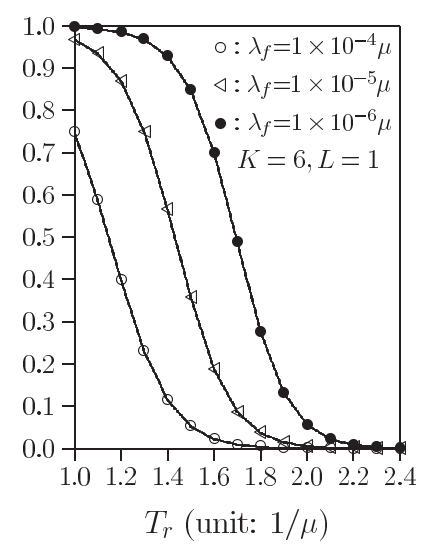

(b) Effect of $\lambda_{f}$
Fig. 3. Effects of $T_{r}, L$ and $\lambda_{f}$ on $\alpha\left(\lambda_{c}=\mu / 18\right)$

Eq. (35) says that the $m$-th GTP' message arrival is the $(j+1)$ th Echo message, and there are $m-j-1$ charging packets occurring in period $\tau(m)$, which has the Poisson distribution with parameter $\lambda_{c}$. For a given $\tau_{0}$ and $m>0$, the expected value of $\tau(m)$ is

$$
\begin{aligned}
& E\left[\tau(m) \mid \tau_{0}\right]=\int_{\tau(m)=0}^{\infty}\left[1-F_{m}\left(\tau(m) \mid \tau_{0}\right)\right] d \tau(m) \\
= & \left(\frac{1}{\lambda_{c}}\right) \sum_{i=0}^{m-1}\left\{1-e^{-\lambda_{c}\left[\tau_{0}+(m-i-1) T_{e}\right]}\right\} \\
& \times\left\{\sum_{j=0}^{i} \frac{\left\{\lambda_{c}\left[\tau_{0}+(m-i-1) T_{e}\right]\right\}^{j}}{j !}\right\}
\end{aligned}
$$

Since $t_{f}$ is a random observer of the inter-Echo arrival times, $\tau_{0}$ is uniformly distributed over $\left(0, T_{e}\right]$. From (11), (31) and (36), the expected value of $E\left[\tau_{d}\right]$ is expressed as

$$
\begin{aligned}
E\left[\tau_{d}\right]= & E\left[\tau_{d} \mid m>0\right] \operatorname{Pr}[m>0]+E\left[\tau_{d} \mid m=0\right] \operatorname{Pr}[m=0] \\
= & \left(\frac{1}{T_{e}}\right) \sum_{m=1}^{K} \int_{\tau_{0}=0}^{T_{e}}\left(E\left[\tau(m) \mid \tau_{0}\right]+L T_{r}\right) \\
& \quad \times \operatorname{Pr}\left[N_{K}\left(t_{f}+L T_{r}\right)=K-m \mid \tau_{0}\right] d \tau_{0} \\
& \quad+E\left[\tau_{d} \mid m=0\right] \operatorname{Pr}[m=0]
\end{aligned}
$$

where $E\left[\tau_{d} \mid m=0\right]$ and $\operatorname{Pr}[m=0]$ are derived in [12].

The analytic model developed in this paper is validated against the simulation experiments. The discrepancies between analytic analysis (specifically, Eqs. (9) and (37)) and simulation are within $3 \%$ in most cases. The simulation technique used in this paper is similar to the one described in [6], and the details are omitted.

\section{NUMERICAL EXAMPLES}

Based on the analytic model developed in the previous section, we show how $K, L$ and $T_{r}$ affect the probability $\alpha$ of false failure detection and the expected time $E\left[\tau_{d}\right]$ of true failure detection. We assume that the round-trip transmission delay $t_{r}$ between a GSN and a CG has a hyper-Erlang distribution with the expected value $1 / \mu=\sum_{i=1}^{M} \beta_{i} / \mu_{i}$ and 
the distribution function

$$
F_{r}\left(t_{r}\right)=1-\sum_{i=1}^{M} \beta_{i}\left\{\sum_{j=0}^{m_{i}-1}\left[\frac{\left(m_{i} \mu_{i} t_{r}\right)^{j}}{j !}\right] e^{-m_{i} \mu_{i} t_{r}}\right\}
$$

where $M, m_{1}, m_{2}, \ldots, m_{M}$ are nonnegative integers, $\mu_{i}>0$, $\beta_{i}>0$, and $\sum_{i=1}^{M} \beta_{i}=1$. The hyper-Erlang distribution is selected because this distribution has been proven as a good approximation to many distributions as well as measured data [4], [5]. From (5) and (38)

$$
p=\left\{\sum_{i=1}^{M} \beta_{i}\left\{\sum_{j=0}^{m_{i}-1}\left[\frac{\left(m_{i} \mu_{i} T_{r}\right)^{j}}{j !}\right] e^{-m_{i} \mu_{i} T_{r}}\right\}\right\}^{L}
$$

In our study, the input parameters $\lambda_{c}, \lambda_{f}, T_{r}$ and the output measure $E\left[\tau_{d}\right]$ are normalized by the mean $1 / \mu$ of the roundtrip transmission delay. For purposes of demonstration, we consider $t_{r}$ with a 2-Erlang distribution and $K L=6$. The Echo message arrivals is a deterministic stream with fixed interval $T_{e}=18 / \mu$.

\section{A. Effects of input parameters on $\alpha$}

Based on (9), Fig. 3(a) plots $\alpha$ against $T_{r}$ and the $(K, L)$ pair, where $\lambda_{c}=\mu / 18$ and $\lambda_{f}=1 \times 10^{-5} \mu$. It is trivial that $\alpha$ is a decreasing function of $T_{r}$. The non-trivial result is that Fig. 3(a) quantitatively indicates how the $T_{r}$ value affects $\alpha$. When $T_{r}<2 / \mu$, increases $T_{r}$ significantly reduces $\alpha$. On the other hand, when $T_{r}>2 / \mu$, increasing $T_{r}$ does not improve the performance. Also, for small $T_{r}, L=1$ outperforms other $L$ setups. Same effect is observed for other $\lambda_{c}$ values. When $T_{r}$ is large, the $L$ (and thus $K$ ) values have same impact on $\alpha$.

Fig. 3(b) plots $\alpha$ as a function of $T_{r}$ and $\lambda_{f}$, where $K=6$, $L=1$ and $\lambda_{c}=\mu / 18$. This figure shows that $\alpha$ increases as $\lambda_{f}$ decreases. When $\lambda_{f}$ decreases (i.e., the system reliability improves but the transmission delay distribution remains the same as before), the GTP' connection lifetime becomes longer. Therefore, the opportunity for false failure detection increases. For $T_{r}=1.6 / \mu$, when the system reliability increases from $\lambda_{f}=1 \times 10^{-5} \mu$ to $\lambda_{f}=1 \times 10^{-6} \mu, \alpha$ increases by 2.72 times. This effect becomes insignificant when $T_{r}$ is large (e.g., $\left.T_{r}>2.2 / \mu\right)$.

Fig. 4(a) plots $\alpha$ as a function of $T_{r}$ and $\lambda_{c}$, where $K=6$, $L=1$ and $\lambda_{f}=1 \times 10^{-5} \mu$. This figure shows that $\alpha$ increases as $\lambda_{c}$ increases. When there are more GTP' message arrivals, it is more likely that false failure detection occurs. This effect is insignificant when $T_{r}$ becomes large (e.g., $T_{r}>2 / \mu$ ).

\section{B. Effects of input parameters on $E\left[\tau_{d}\right]$}

Based on (37), Fig. 4(b) plots $E\left[\tau_{d}\right]$ as a function of $T_{r}$ and $\lambda_{c}$, where $K=6, L=1$. This figure shows that $E\left[\tau_{d}\right]$ significantly increases as $\lambda_{c}$ decreases.

Figs. 5(a) and 5(b) plot $E\left[\tau_{d}\right]$ as functions of $T_{r}$ and the $(K, L)$ pair, where $\lambda_{c}=\mu$ and $\lambda_{c}=\mu / 36$, respectively. These figures show that $E\left[\tau_{d}\right]$ is an increasing function of $T_{r}$ and $E\left[\tau_{d}\right]$ is more sensitive to the change of $T_{r}$ when $L$ is large than when $L$ is small. When $\lambda_{c}=\mu, E\left[\tau_{d}\right]$ is larger for $L=6$ than for $L=1$. When $\lambda_{c}=\mu / 36$, the opposite results

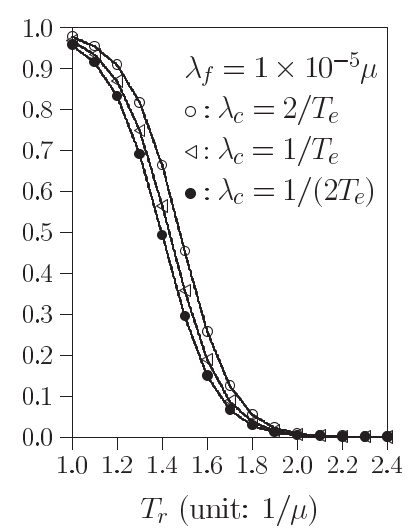

(a) $\alpha$

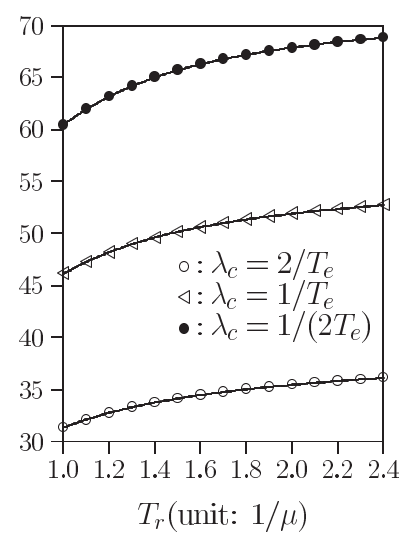

(b) $E\left[\tau_{d}\right]$ (unit $1 / \mu$ )
Fig. 4. Effects of $T_{r}$ and $\lambda_{c}(K=6, L=1)$

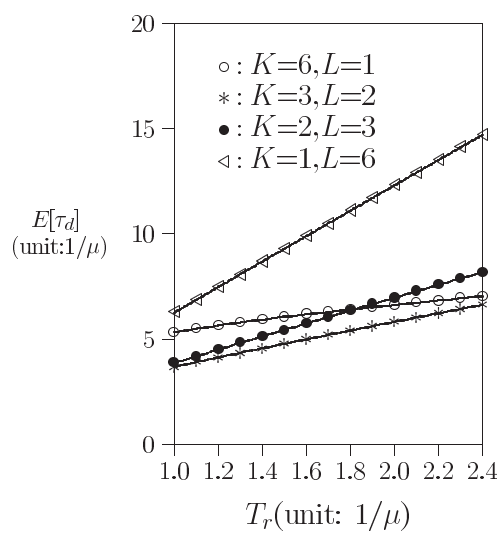

(a) $\lambda_{c}=\mu$

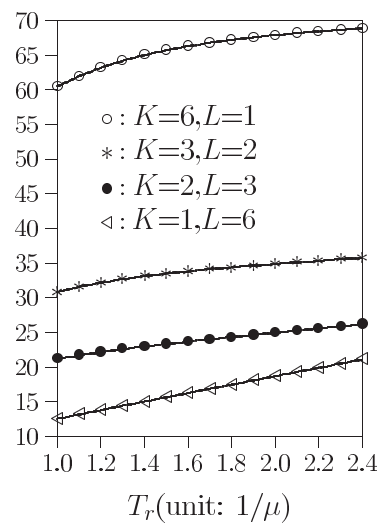

(b) $\lambda_{c}=\mu / 36$
Fig. 5. Effects of $T_{r}$ and $L$ on $E\left[\tau_{d}\right]$

are observed. This phenomenon can be explained as follows. Without loss of generality, assume that $t_{a, 1} \geq t_{f}$. Consider an extreme case that $\lambda_{c}$ is very large, and many GTP' charging packets arrive in a very short period $\left(t^{\prime}, t^{\prime}+d t^{\prime}\right)$ where $t^{\prime} \geq t_{f}$. For $L=1(K=6), t_{a, 6} \approx t^{\prime}$ and $t_{d, 6} \approx t^{\prime}+T_{r}$. Therefore, the true failure detection time is $t_{d} \approx t^{\prime}+T_{r}$. For $L=6(K=$ 1 ), we have $t_{a, 1} \approx t^{\prime}$, but the true failure detection time is $t_{d}=t_{d, 1} \approx t^{\prime}+6 T_{r}$. Therefore, $E\left[\tau_{d}\right]$ is larger for $L=6$ than for $L=1$ in Fig. 5(a).

On the other hand, when $\lambda_{c}$ is small, the charging packets rarely occur in a short period, and it is likely that $t_{a, i+1}-t_{a, i}>$ $T_{r}$ (for $i>0$ ). For $L=1$, the failure is detected at $t_{a, 6}+T_{r}$. For $L=6$, the failure is detected at $t_{a, 1}+6 T_{r}$. Under the situation that $t_{a, i+1}-t_{a, i}>T_{r}$, we have $t_{a, 6}-t_{a, 1}>5 T_{r}$. Therefore, we expect that $E\left[\tau_{d}\right]$ is smaller for $L=6$ than for $L=1$ in Fig. 5(b).

\section{CONCLUSIONS}

In UMTS, the GTP' protocol is used to deliver the CDRs from GSNs to CGs. To ensure that the mobile operator receives the charging information, availability for the charging system is essential. One of the most important issues on GTP' availability is connection failure detection. This paper studied the GTP' connection failure detection mechanism specified in 3GPP TS 29.060 and 3GPP TS 32.215. The output measures 
considered are the false failure detection probability $\alpha$ and the expected time $E\left[\tau_{d}\right]$ of true failure detection. We proposed an analytic model to investigate how these two output measures are affected by input parameters including the Charging Packet Ack Wait Time $T_{r}$, the Maximum Number $L$ of Charging Packet Tries and the Maximum Number $K$ of Unsuccessful Deliveries. We make the following observations.

- When $T_{r}$ is small, increasing $T_{r}$ reduces $\alpha$ significantly. When $T_{r}$ is sufficiently large, increasing $T_{r}$ only has insignificant impact on $\alpha$. On the other hand, increasing $T_{r}$ always non-negligibly increases $E\left[\tau_{d}\right]$.

- $\alpha$ increases as the charging packet arrival rate $\lambda_{c}$ increases. This effect is insignificant when $T_{r}$ becomes large. On the other hand, the effects of $\lambda_{c}$ on $E\left[\tau_{d}\right]$ are not the same for different $(K, L)$ setups. In our examples, when $\lambda_{c}$ is large, $E\left[\tau_{d}\right]$ is larger for $L=6$ than for $L=1$. When $\lambda_{c}$ is small, $E\left[\tau_{d}\right]$ is smaller for $L=6$ than for $L=1$. Therefore, the effects of $\lambda_{c}$ should be considered when we select the $L$ value.

In summary, the network operator can select the appropriate $T_{r}, L$ and $K$ values for various traffic conditions based on our study.

\section{REFERENCES}

[1] 3rd Generation Partnership Project, Technical Specification Group Services and Systems Aspects, "Architectural Requirements for Release" 1999 (Release 1999), 3G TS 23.121 version 3.6.0 (2002-06), 2002.

[2] 3rd Generation Partnership Project, Technical Specification Group Core Network, General Packet Radio Service (GPRS), "GPRS Tunneling Protocol (GTP) across the Gn and Gp Interface" (Release 5), 3G TS 29.060 version 5.9 .0 (2004-03), 2004.

[3] 3rd Generation Partnership Project, Technical Specification Group Services and Systems Aspects, Telecommunication management, Charging management, "Charging data description for the Packet Switched (PS) domain" (Release 5), 3G TS 32.215 version 5.5.0 (2003-12), 2003.

[4] Y. Fang, and I. Chlamtac, "Teletraffic analysis and mobility modeling for PCS networks,"IEEE Trans. Commun., vol. 47, no.7, pp. 1062-1072, July 1999.

[5] F. P. Kelly, Reversibility And Stochastic Networks. John Wiley \& Sons, 1979.

[6] Y.-B. Lin, and Y.-K. Chen, "Reducing authentication signaling traffic in third generation mobile network," IEEE Trans. Wireless Commun., vol.2, no.3, pp. 493-501, May 2003.

[7] Y.-B. Lin and I. Chlamtac, Wireless and Mobile Network Architectures. JohnWiley \& Sons, 2001.
[8] Y.-B. Lin, Y.-R. Haung, A.-C. Pang, and I. Chlamtac, "All-IP approach for UMTS third generation mobile networks," IEEE Network, vol. 16, no.5, pp. 8-19, Sept. 2002.

[9] R. G. Gallager, Discrete Stochastic Processes. Kluwer Academic Publishers, 1999.

[10] S. M. Ross, A First Course in Probability. Prentice Hall, 2001.

[11] S. M. Ross, Stochastic processes. JohnWiley \& Sons, 1996.

[12] H.-N. Hung, Y.-B. Lin, N.-F. Peng, and S.-I. Sou, Connection Failure Detection Mechanism of UMTS Charging Protocol. Technical Report, 2004.

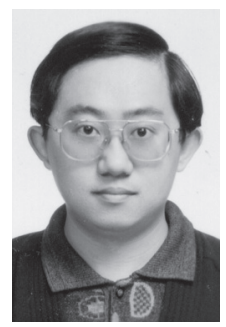

Hui-Nien Hung received the B.S.Math. degree from National Taiwan University, Taiwan, in 1989, the M.S.Math. degree from National Tsin-Hua University, Taiwan, in 1991, and the Ph.D. degree in Statistics from The University of Chicago in 1996. He is a Professor at the Institute of Statistics, National Chiao Tung University, Taiwan. His current research interests include applied probability, financial calculus, bioinformatics, statistical inference, statistical computing and industrial statistics.

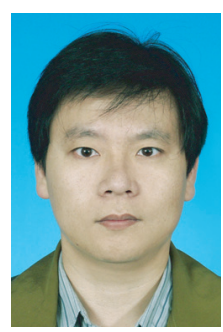

Yi-Bing Lin (M'95-SM'95-F'03) received the B.S.E.E. degree from the National Cheng Kung University in 1983 and the Ph.D. degree in computer science from the University of Washington in 1990. $\mathrm{He}$ is chair professor in the Department of Computer Science and Information Engineering (CSIE), National Chiao Tung University (NCTU). Dr. Lin is a fellow of the IEEE and the ACM.

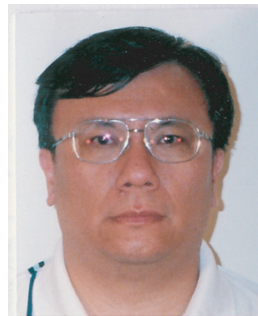

Nan-Fu Peng received the B.S. degree in the applied mathematics from National Taiwan University, Hsinchu, Taiwan, R.O.C., in 1981, and the Ph.D. degree in statistics from The Ohio State University, Columbus, in 1989. He is currently an Associate Professor with the Institute of Statistics, National Chiao Tung University. His research interests include Markov chains, population dynamics, and the queueing theory.

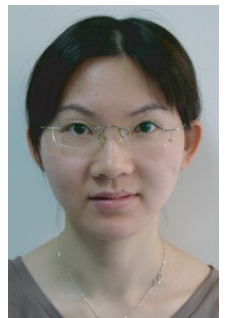

Sok-Ian Sou received the B.S.CSIE. and M.S.CSIE degrees from National Chiao Tung University (NCTU), Taiwan, in 1997 and 2004, respectively. She is currently working toward the Ph.D. degree at NCTU. Her current research interests include personal communications services network, Voice over IP technology and performance modeling. 